\title{
Characterization of a Naturally Occurring Mutation (Ile-12 to Thr) Close to Prosthetic Group FAD in Human Dihydrolipoamide Dehydrogenase
}

\author{
Lin Yuan, Young-Joon Cho, ${ }^{\dagger}$ and Hakjung Kim*
}

\author{
Department of Chemistry and Department of Holectlar Biologv, College of Natural Science, Daegu Lninersity, \\ Kvoungsan 7/2-714, Korea. E-mail: hjkimáadaeguackr \\ Received December 8, 2008, Accepted February 18, 2009
}

Key Words: Dihydrolipoamide delydrogenase. Pyridine nucleotide-disulfide oxidoreductase. Site-directed mutagenesis. Dihydrolipoamide deficiency $\alpha$-Keto acid dehỳdrogenase complexes

Dilyddrolipoamide delyydrogenase (E3) (dilyydrolipoamide: $\mathrm{NAD}^{-}$oxidoreductase: $\mathrm{EC}$ 1.8.1.4) is a common component in three $\alpha$-keto acid dehydrogenase complexes (py ruvate, $\alpha$ ketoglutarate and branched-chain $\alpha$-keto acid dehydrogenase complexes) ${ }^{1}$ and the glycine cleavage sy stem. ${ }^{2}$ It catalyzes the reoxidation of the dilydrolipoyl prosthetic group attached to the lysyl residue(s) of the acyltransferase components of the three $\alpha$-keto acid dehydrogenase complexes and to the hydrogen-carrier protein of the glycine cleavage system. It is a homodimeric flavoenzy'me containing one $\mathrm{FAD}$ at each subunit. ${ }^{3}$ Patients with E3 deficiency have various clinical symptoms. ranging from severe neurological defects such as Leigh syndrome to less severe illness in childhood such as exertional fatigue. One substitution mutation of Ile- 12 to $\mathrm{Thr}$ was found in two male second cousins with E3 deficiency. ${ }^{4}$

Site-directed mutagenesis method has been a useful tool for the structure-function study of human $E 3$ and other proteins. ${ }^{5-10}$ By the site-specific mutations of Ile-12 to Thr. the effects of this naturally occurring mutation in human E3 structure and function were examined. The site-directed mutagenesis was performed using a mutagenesis kit according to the provided protocol. PCRs were carried out using the human E3 expression vector pPROEX-1:E3 as a template in a programmable PCR machine with the appropriate primers. Whole DNA sequence of human E3 coding region was sequenced to verify the integrity of DNA sequences other than the anticipated mutation.

The mutant (Ile-12 to Thr) was expressed in $E$. coll by IPTG $(1 \mathrm{mM})$ induction. The purification of the mutant was performed using nickel affinity column according to the provided protocol. The mutant was eluted with the elution buffer containing $250 \mathrm{mM}$ imidazole. SDS-PAGE showed that the mutant was highly purified. Its molecular weight was the same to that of wild-type human E3 on the SDS-PAGE gel. E3 assay was performed at $37^{\circ} \mathrm{C}$ in a $50 \mathrm{mM}$ potassium

Table 1. Steady state kinetic parameters of both mutant (Ile-12 to Thr) and wild-type human E3s. E3 assay was pertomed at $37^{\circ} \mathrm{C}$ in a $50 \mathrm{mM}$ potassium phosphate bufter $(\mathrm{pH} 8.0)$ containing $1.5 \mathrm{mM}$ EDTA with variable concentrations of the substrates.

\begin{tabular}{lccc}
\hline Humant $\mathrm{E} 3 \mathrm{~s}$ & $\begin{array}{c}k_{\text {cot }} \\
\left(\mathrm{s}^{-1}\right)\end{array}$ & $\begin{array}{c}K_{m} \text { for DHL } \\
(\mathrm{mM})\end{array}$ & $\begin{array}{c}K_{m s} \text { for NAD } \\
(\mathrm{mM})\end{array}$ \\
\hline $\begin{array}{l}\text { Wild-type } \\
\text { Mutant }\end{array}$ & 899 & 0.64 & 0.19 \\
\hline
\end{tabular}

phosplate buffer $(\mathrm{pH} 8.0)$ containing $1.5 \mathrm{mM}$ EDTA with variable concentrations of the substrates. dihydrolipoamide and $\mathrm{NAD}^{-}$. The steady state kinetic parameters were determined using the SigmaPlot Enzyme Kinetics Module (Systat Software Inc.. San Jose. USA). Table 1 shows the kinetic parameters of both mutant and wild-type human E3s. The $k_{\text {sut }}$ value was similar to that of normal human $\mathrm{E} 3$. The $K_{m}$ value for dihydrolipoamide was slightly lower than that of normal human E3. However. the $K_{m}$ value for $\mathrm{NAD}^{-}$was about 2.4-fold larger than that of normal human E3, indicating that the mutation makes the enzyme less efficient to $\mathrm{NAD}^{-}$. In a

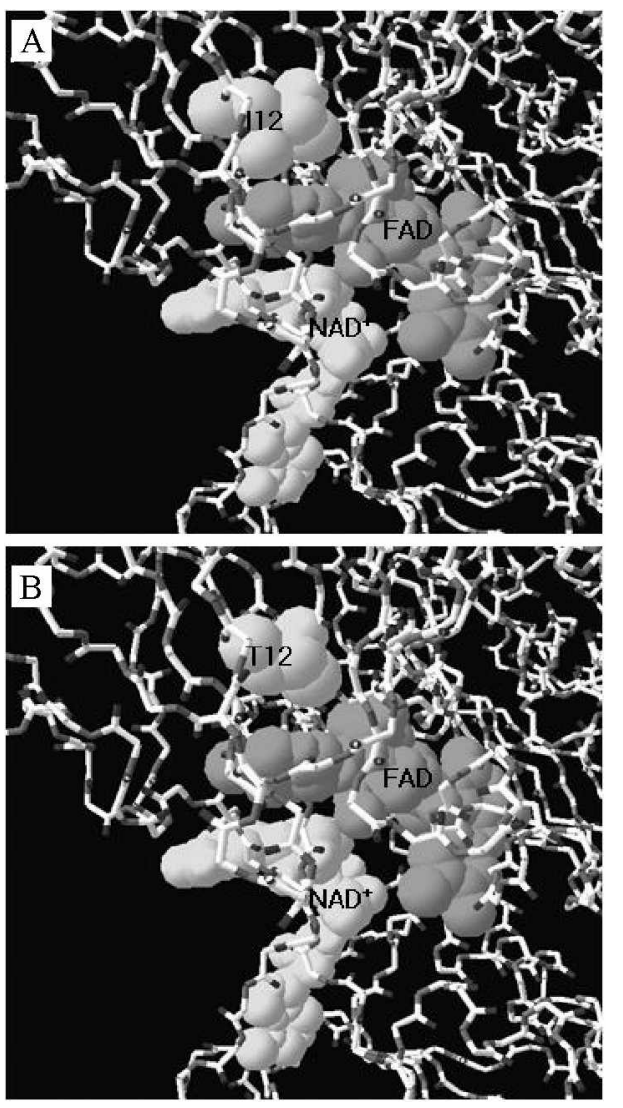

Figure 1. The location of Ile-12 in human E3 structure. The f AD), $\mathrm{NAD}^{-}$and residue-12 are shown in space-filled structures and other residues are shown in backbone structures. A: Before the mutation, $\mathrm{B}$. After the mutation (Ile-12 to Thr 

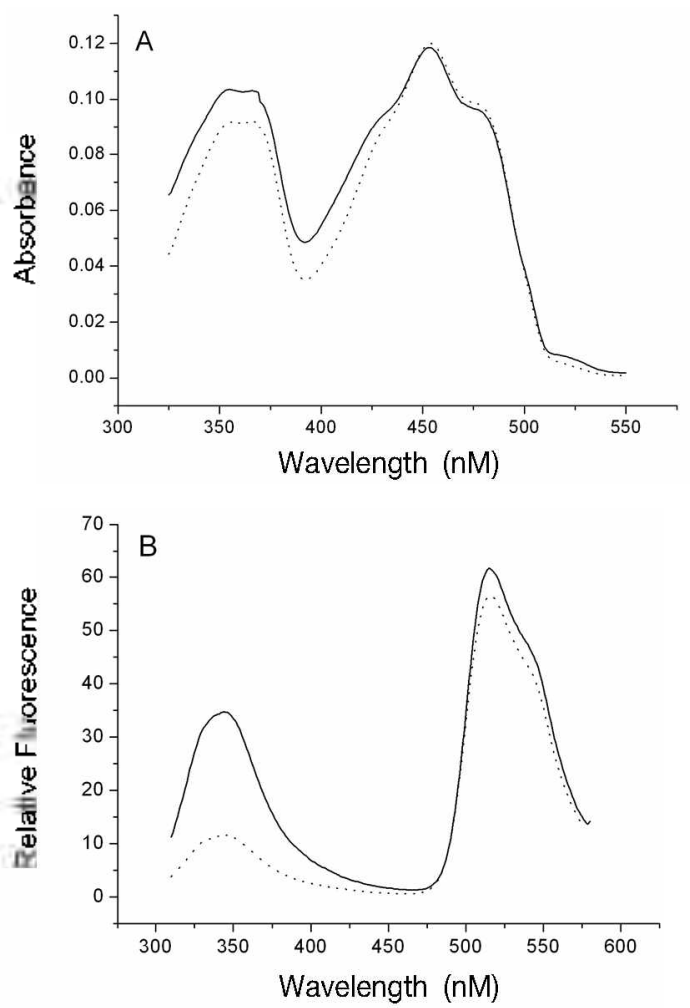

Figure 2. Spectroscopic properties of both mutant (Ile-12 to Thr) and wild-type humann E3s. A: UV-visible spectra of the mutant (solid line) and wild-type (dotted line) human E3s. B; Fluorescence spectra of mutant (solid line) and wild-type (dotted line) human E3s.

human E3 structure. Ile- 12 is located close to prosthetic group $\mathrm{FAD}$ as shown in Figure 1 . It is part of a $\beta$-sheet secondary structure in the FAD domain. The amino acid volume of Ile is $166.7 \mathrm{~A}^{3}$ while that of Thr is $116.1 \mathrm{~A}^{3}$. The mutation will give a vacancy of $50.6 \mathrm{~A}^{3}$ at the residue-12. The $\mathrm{OH}$ group of Thr- 12 side chain can form a new hydrogen bond with the $\mathrm{CO}$ group of Leu-145 backbone. The mutation of Ile-12, a large hydrophobic amino acid. to Thr. a smaller hydrophilic amino acid. could cause structural changes in the local structure of this region. These stnictural changes could be responsible for the alterations in kinetic parameters of the mutant

To examine any structural changes occurring in the mutant, UV-visible absorption and fluorescence spectroscopies were performed. Because $\mathrm{E} 3$ contains FAD as a prosthetic group. human E3 shows a characteristic UV-visible absorption spectrum of flayoproteins as shown in Figure 2A. The overall shape of the mutant spectrum (solid line) was similar to that of the wild-type human E3 spectnum (dotted line). However. noticeable differences can be observed in the peak at around $350 \mathrm{~nm}$. The peak of the mutant was slightly higher than that of the wild-type human E3. In case of the mutant (solid line), the ratio of the absorbance value of the second peak (at around $455 \mathrm{~nm}$ ) to the absorbance value of the first peak (at around $350 \mathrm{run}$ ) was about 1.15 , which was slightly lower than the ratio (about 1.30 ) in case of the wild-type enzyme (dotted line). These differences in the UV-visible spectrum of the mutant indicated that stnictural changes could occur in the enzyme. More evidence of the structural changes in the mutant came from the fluorescence study. When enzymes were excited at $296 \mathrm{lum}$. two fluorescence enissions were observed for both mutant and wild-type E3s. as shown in Figure 2B. The first emission from $305 \mathrm{~nm}$ to $400 \mathrm{~nm}$ was mainly $\operatorname{Tr} p$ fluorescence. The second enission from $480 \mathrm{~nm}$ to over $550 \mathrm{~nm}$ was FAD fluorescence. In human E3. the Trp fluorescence was quenched due to fluorescence resonance energy transfer from $\operatorname{Trp}$ to $\mathrm{FAD}$. When the fluorescence spectra of E3s were compared. a noticeable difference was found in the ratio between relative intensities of the first and second fluorescence emissions. The ratio (about 1.8 ) between relative intensities of the first and second fluorescence emissions of the mutant (solid line) was much smaller than that (about 4.9) of the wild-type enzyme (dotted line). This was mainly due to the increase in the first fluorescence of $\operatorname{Trp}$ residues. This also indicated that the energy transfer from Trp to FAD was disturbed in the Thr-12 nutant. The structural changes due to the mutation of Ile-12 to Thr could have affected the structure of human E3 so that the Trp fluorescence was increased and the fluorescence resonance energy transfer from $T r p$ residues to FAD was interfered with.

In this study, the effects of a naturally occurring nutation (Ile-12 to Thr) in human E3 structure and function were examined using site-directed nutagenesis. E3 activity measurement and spectroscopic methods. The mutation of Ile-12 to Thr in human E3 resulted in the structural changes which altered the UV-visible spectrum, and increased the Trp fluorescence. and interfered with the efficient fluorescence resonance energy transfer from $\operatorname{Trp}$ residues to FAD. These structural changes could affect the kinetic parameters of the nutant, indicating that the mutant became less efficient to $\mathrm{NAD}^{-}$. These findings indicated that the conservation of Ile- 12 residue in human $E 3$ was inportant to its structure and function.

Aclonowledgments. The authors thank Dr. Mulchand S. Patel (University at Buffalo. the State University of New York) for a generous gift of an $E$. coli XL l-Blue containing a human E3 expression vector. The authors are grateful to Dr. Tai Jong Kang (Daegu University) for providing a fluorometer. This research was supported in part by the Daegu University Research Grant. 2006.

\section{Refeiences}

1. Reed, L. J. Acc. Chem. Res. 1974, 7, 40.

2. Walker, J. L.; Oliver, D. T. J. Biol. Chent 1986, 261, 2214

3. Brautigam, C. A.; Chuang, J. L.; Tomchick, D. R.; Machius, M: Chuang, D. T. J. Mol. Biol. 2005, 350, 543.

4. Cameron, J. M.: Levandovskiy, V.; Mackay, N.: Raman, I.; Renaud, D. L.; Clarke, T. T.; Feigenbaum, A.; Elpeleg, O.; Robinson, B. H. Am. J. Wed Genet 2006, 140, 1542 .

5. Kim, H.; Patel, M. S. J. Biol. Chent 1992, 267, 5128

6. Kim, H. Bull. Korean Chem. Soc. 2007, $28,907$.

7. Karm, M.; Shm, M.-Y; Km, I.: Chol, K.-I.: Km, I.-R:; Chol, J.-D.: Yoon, M.-Y. Bull. Korean Chent Soc. 2006, 27, 549.

8. Koh, J.-U; Cho, H.-Y.; Kong, K.-H. Bull. Korean Chem. Soc. 2007, 28, 772 .

9. Park, S. H.; Km, B. G.: Lee, S. H.; Lm, Y.; Cheong, Y.: Ahn, J.-H. Bull. Korem Chent. Soc. 2007, 28, 2248.

10. Quven, D. V.; Ha, S. C.; Kim, D; Lee, S.; Park, S.-T.; Kim, K. K; Kim. Y.-G. Bull Korean Chem Soc. 2007. 28.2539 